\title{
Highly Dissimilar Behaviors Mediated by a Multifunctional Network in the Marine Mollusk Tritonia diomedea
}

\author{
Ion R. Popescu and William N. Frost \\ Department of Cell Biology and Anatomy, Finch University of Health Sciences, The Chicago Medical School, North \\ Chicago, Illinois 60064
}

\begin{abstract}
Several motor networks have now been found to be multifunctional, in which one group of neurons participates in the generation of multiple behavioral motor programs. Not surprisingly, the behaviors involved are frequently closely related, often using the same or similar muscle groups. Here we describe an interneuronal network in the marine mollusk Tritonia diomedea that is involved in producing two highly dissimilar behaviors, rhythmic, muscle-based escape swimming and nonrhythmic, cilia-mediated crawling. Several observations support this conclusion. First, the dorsal swim interneurons (DSIs) of the swim central pattern generator (CPG) directly excite Pedal neuron 21 (Pd21) and Pd5, the only identified cilia-activating efferent neurons in Tritonia. Second, stimulation of a single DSI elicits beating of the foot cilia in semi-intact preparations and crawling in intact animal treadmill preparations. Third, the DSIs fire at an elevated rate for nearly $1 \mathrm{hr}$ after a swim motor program, which
\end{abstract}

correlates reasonably well with the period freely behaving animals were found to crawl after they swam. Fourth, silencing the tonically active DSIs after a swim motor program substantially reduces or eliminates ongoing cilia neuron firing, indicating that the DSIs are major contributors to the synaptic input driving these cells. Finally, all of the other swim CPG neurons also connect to the cilia neurons, most monosynaptically. Taken together, these observations indicate that the Tritonia swim CPG network participates in producing both escape swimming and crawling. Given the extreme differences between these behaviors--rhythmic versus tonic, muscular versus ciliary, and brief versus prolonged-these findings reveal a striking versatility for a small multifunctional network.

Key words: Tritonia; multifunctional network; central pattern generator; cilia; locomotion; mollusk; CPG; evolution; multifunctional neuron
An emerging principle of motor control is that some neural networks mediate multiple behaviors (Getting, 1989; Pearson, 1993; Morton and Chiel, 1994; Dickinson, 1995; Marder and Calabrese, 1996; Kupfermann and Weiss, 2001). Examples from invertebrates include networks mediating multiple feeding rhythms in crustacea (Meyrand et al., 1991; Weimann and Marder, 1994), swimming rhythms in jellyfish (Mackie and Meech, 1985), types of siphon withdrawal in Aplysia (Frost and Kandel, 1995), and leg movements in stick insects (Kittmann et al., 1996). Multifunctional networks have also been suggested to underlie certain vertebrate behaviors, such as the generation of different breathing patterns (Lieske et al., 2000) and the ingestion and rejection of food (Travers et al., 2000).

The existence of such networks prompts a number of questions. How are the different motor programs of such networks selectively activated? Can multifunctional networks incorporate modulation specific to one behavior, as might occur in learning, without altering the other behaviors mediated by the same network? Just how dissimilar can behaviors be and still be mediated by a single neuronal network? Are multifunctional networks relatively rare, or are they a widespread feature of most nervous systems? Such questions can most effectively be addressed in

Received Aug. 6, 2001; revised Dec. 10, 2001; accepted Dec. 18, 2001.

This research was supported by National Institutes of Health Grant NS36500. We thank Lise Eliot for comments on this manuscript and Friday Harbor Laboratories for use of their facilities during the summer.

Correspondence should be addressed to William N. Frost, Department of Cell Biology and Anatomy, Finch University of Health Sciences, The Chicago Medical School, 3333 Green Bay Road, North Chicago, IL 60064. E-mail: wfrost@finchcms.edu. Copyright (C) 2002 Society for Neuroscience $0270-6474 / 02 / 221985-09 \$ 15.00 / 0$ preparations allowing detailed electrophysiological dissection of the individual components of multifunctional networks.

The escape swim network of the marine mollusk Tritonia diomedea has previously been suggested to be multifunctional for swimming and reflexive withdrawal (Getting and Dekin, 1985b; Getting, 1989), with its role in swimming particularly well documented (Getting, 1983a). After the swim, Tritonia crawl for a period (Audesirk and Audesirk, 1980). In contrast to swimming, crawling is a nonmuscular, nonrhythmic, gliding form of locomotion propelled by the beating of foot cilia against the substrate (Audesirk, 1978a). Because swimming and crawling are so dissimilar, we did not initially suspect them to be mediated by the same network.

Although no role for the swim network in crawling had been suggested before this study, certain previous findings led us to suspect such a role. First, this laboratory and others had noted that one particular group of central pattern generator (CPG) neurons, the dorsal swim interneurons (DSIs), fire tonically at an elevated rate for several minutes after swim motor programs elicited in isolated brain preparations (Lennard et al., 1980; Katz et al., 1994). Intact animals had previously been observed to crawl during the postswim period (Audesirk et al., 1979). Second, the DSIs contain serotonin (Katz et al., 1994; McClellan et al., 1994; Fickbohm and Katz, 2000), a transmitter known to activate the locomotor cilia in reduced preparations (Audesirk et al., 1979; Willows et al., 1997) and crawling in intact animals (McClellan et al., 1994). Third, the cerebral neuron 2 (C2) neurons of the swim CPG were known to make direct synaptic connections onto Pedal neuron $21(\mathrm{Pd} 21)$ and $\mathrm{Pd} 5$ (Snow, 1982), cells that other studies had shown to be cilia-activating efferent neurons (Audesirk, 
1978a; Popescu and Willows, 1999). In the present study, we attempted to directly test whether the swim CPG has a role in crawling, and we conclude that it does so; indeed, because the animal spends much more time crawling than swimming, the excitatory and inhibitory control of crawling may turn out to be the predominant operating mode of the network.

\section{MATERIALS AND METHODS}

Behavior. The duration of postswim crawling was measured in an $88 \times 57$ $\mathrm{cm}$ test arena created within a 120 gallon, $10^{\circ} \mathrm{C}$ recirculating artificial seawater aquarium. A plastic mesh (grid dimensions, $1.5 \times 2.0 \mathrm{~mm}$ ) fixed to the arena floor provided a background against which crawling could be visually assessed. During each observation period, animals were scored as crawling if the tip of the tail was observed to progress at least 1 grid unit along the substrate during a $15 \mathrm{sec}$ period. Any animal whose foot was not in contact with the substrate during the observation period was not scored for that session rather than scored as not crawling. This was because such animals are often observed to have a steady stream of debris particles moving along the upturned foot, indicating that they are in "crawling mode."

Isolated brain preparation. For most electrophysiology experiments, the brain, consisting of the fused cerebral-pleural ganglia and the pedal ganglia (with the pedal-pedal commissure cut), was dissected from the animal and pinned dorsal side-up in a Sylgard-lined recording chamber perfused with normal saline at $4-6^{\circ} \mathrm{C}$. Normal saline consisted of (in $\mathrm{mM}$ ): $420 \mathrm{NaCl}, 10 \mathrm{KCl}, 10 \mathrm{CaCl}_{2}, 50 \mathrm{MgCl}_{2}, 10 \mathrm{HEPES}$, pH 7.6, and 11 D-glucose. All procedures used stainless steel minuten pins (0.1 and 0.2 $\mathrm{mm}$ thickness) to stabilize the nervous system for recording. After dissecting away the connective tissue sheath covering the cerebralpleural ganglia, a polyethylene suction electrode was attached to left or right pedal nerve 3 (PdN3; for nomenclature, see Willows et al., 1973). The perfusion temperature was then raised to $11^{\circ} \mathrm{C}$, and the preparation was rested for a minimum of $3 \mathrm{hr}$. The lower initial saline temperature served to minimize neural activity during desheathing.

Intracellular recordings were made with $15-40 \mathrm{M} \Omega$ electrodes filled with $3 \mathrm{M} \mathrm{KCl}$ or $3 \mathrm{~m} \mathrm{~K}$-acetate. Neurons were identified on the basis of their location, size, color, synaptic connections with other identified neurons, and activity during the swim motor program, as described previously (for Pd5 and Pd21, see Willows et al., 1973; Audesirk, 1978b; for CPG neurons, see Getting, 1983a). Swim motor programs were elicited by applying a $10 \mathrm{~Hz}, 2 \mathrm{sec}$ train of $5 \mathrm{msec} 10 \mathrm{~V}$ pulses to pedal nerve 3 via a suction electrode. Data were recorded on magnetic tape and analyzed later off-line with a Biopac MP100 data acquisition system.

Synaptic connections were considered direct if presynaptic action potentials produced one-for-one, constant-latency postsynaptic potentials in the postsynaptic neuron that persisted in a high divalent cation solution. This solution consisted of (in mM): $285 \mathrm{NaCl}, 10 \mathrm{KCl}, 25 \mathrm{CaCl}_{2}$, $125 \mathrm{MgCl}_{2}, 10$ HEPES, pH 7.6, and $11 \mathrm{D}$-glucose and has been shown to be effective at reducing the recruitment of polysynaptic pathways in Tritonia (Katz and Frost, 1995b).

Dorsal semi-intact preparation. Animals were anesthetized by injecting $60 \mathrm{ml}$ of a solution composed of half $350 \mathrm{mM} \mathrm{MgCl}_{2}$ and half artificial seawater (Instant Ocean; Aquarium Systems). A recording chamber was used in which the animal could be positioned dorsal side-up, with the brain exposed and stabilized on the Sylgard surface of a 1-cm-diameter post rising from the chamber floor. A thin cylindrical sleeve, containing slits to allow the nerves passage, was raised around the brain; the slits were closed with Vaseline; and the brain and body chambers were perfused separately with saline. The brain chamber was initially perfused at $2^{\circ} \mathrm{C}$, during which the thin sheath enclosing the ganglia was removed to expose the neurons for intracellular recording. Once the neurons were exposed, both brain and body chambers were perfused at $11^{\circ} \mathrm{C}$ for the duration of the experiment.

Ventral semi-intact preparation. This preparation, used for measuring the rate of charcoal particle transport on one side of the foot while driving a contralateral DSI, is identical in methods to the dorsal semiintact preparation, with the following exceptions. After the anesthesia, the animal was placed ventral side-up in a dissection tray filled with $50 \%$ $\mathrm{MgCl}_{2}$ and $50 \%$ artificial seawater originally at $4^{\circ} \mathrm{C}$ but allowed to reach room temperature over the course of the dissection $(\sim 1 \mathrm{hr})$. A midline incision was made in the foot to expose pedal nerve 3, which courses intimate to the viscera. The buccal mass was rotated forward until pedal nerve 3 was exposed rostrally all the way to the brain. The esophagus was cut, and the viscera and buccal mass were removed after the nerve was dissected free from the viscera. Next, an $\sim 4 \mathrm{~cm}$ midline incision was made in the dorsal body wall under the brain. After the cerebral nerves were cut, the brain was pinned dorsal side-up on the Sylgard-covered surface of the post rising from the chamber floor through the dorsal incision.

After resting the preparation overnight at $4^{\circ} \mathrm{C}$, a suspension of charcoal particles (M. Grumbacher Inc., New York, NY) in saline was sprinkled over several square centimeters of foot contralateral to an impaled DSI, about midway to the tail. This area was projected onto a video monitor via a microscope-mounted camera, and particle movement was assessed with the help of a calibrated grid placed over the screen of the monitor. DSI was driven to fire action potentials at $0.5,1$, or $2 \mathrm{~Hz}$ by applying discrete current pulses at these rates through the intracellular electrode. DSI activity and accompanying video of charcoal particle movement were recorded together on videotape with a Vetter 402 data recorder (A. R. Vetter Inc., Rebersburg, PA).

Intact animal treadmill preparation. This preparation (for additional details, see Popescu and Willows, 1999) was used to test whether the DSI neurons could elicit locomotion when stimulated. An incision was made in the dorsal skin overlying the brain, and a set of hooks were attached around the edge of the opening. Threads fixed from the hooks to posts at the top of the chamber walls allowed the opening to be maintained and the animal's position to be stabilized. A small wax-covered manipulatormounted platform was positioned beneath the brain, and minuten pins were placed through the connective tissue to immobilize the brain against the platform. The sheath covering the neurons on the dorsal surface of the fused cerebral and pleural ganglia was then surgically removed to allow intracellular recording from the DSIs. To measure ciliary locomotion, a cylindrical plastic drum was placed into contact with the foot. The end of the drum was covered with a radial pattern of alternating black and white stripes (90 total stripes). Attempted locomotion caused the drum to rotate around a central spindle. A photodiode positioned over a televised image of the stripe pattern converted its rotation into an oscillating voltage signal, the period of which indicated the rate of locomotion.

Data analysis. Results are reported as means \pm SE. Statistical procedures included repeated measures ANOVAs followed by NewmanKeuls post hoc tests and repeated measures $t$ tests with Bonferroniadjusted $p$ values (two-tailed). ANOVA analyses were conducted with the Statistica software package (Statsoft).

\section{RESULTS}

A network diagram depicting the multifunctional Tritonia network is shown in Figure $1 A$. This figure represents the previously known and newly reported synaptic connections and illustrates the involvement of the network in both escape swimming and crawling.

\section{DSI stimulation elicits crawling in an intact animal treadmill preparation}

Previous work noted that after a swim motor program, the DSI neurons of the swim CPG fire tonically at an elevated rate for an undetermined period (Fig. 1B; Lennard et al., 1980; Katz et al., 1994; Frost and Katz, 1996b). Here we sought to determine whether this prolonged DSI firing might be causally related to the episode of crawling that occurs in freely behaving animals after they swim (Audesirk, 1978b; Audesirk and Audesirk, 1980). We began by directly testing whether intracellular DSI stimulation elicits crawling in a treadmill preparation, in which DSI cells could be recorded from and intracellularly stimulated while monitoring ciliary locomotion. In all six attempts in two preparations, driving a single DSI for several seconds at 8-9 $\mathrm{Hz}$ elicited crawling (Fig. 2). In all cases, the locomotion outlasted the DSI firing by tens of seconds.

\section{The DSIs excite cilia-activating neurons with direct excitatory synaptic connections}

Pd21 and Pd5 are presumptive motor neurons that, when driven with intracellular stimulation, excite the locomotor cilia on the animal's foot (Audesirk, 1978a,b; Popescu and Willows, 1999). During the course of the present study, one of us reported that the 

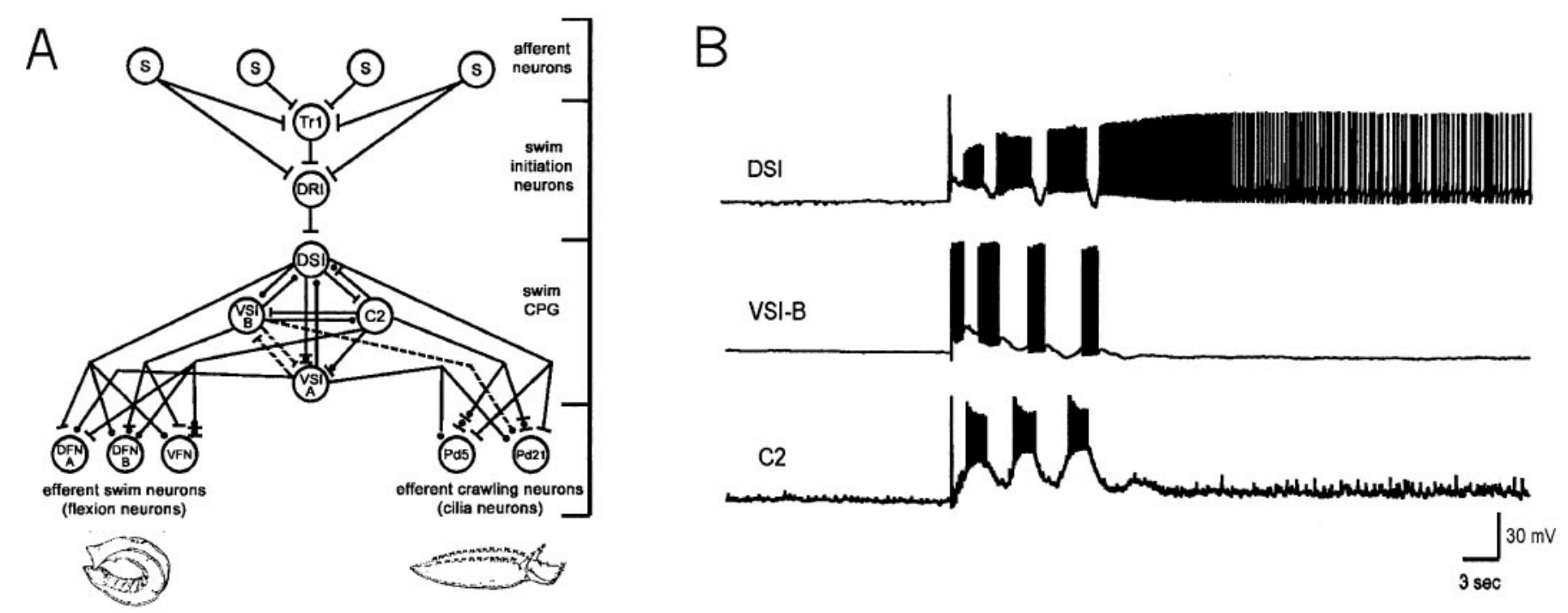

Figure 1. The multifunctional Tritonia network. $A$, Diagram of synaptic connections, showing the afferent neurons $(S)$, pre-CPG interneurons $($ Tr1, $D R I)$, swim CPG neurons (DSI, C2, VSI-A, VSI-B), swim flexion neurons ( $D F N-A, D F N-B, V F N)$, and the locomotion cilia neurons (Pd21, Pd5; (Willows et al.; 1973; Getting, 1983a; Frost and Katz, 1996a; Frost et al.; 2001). The swim CPG consists of just six cells on each side of the brain (3 DSIs, 1 C2, 1 VSI-A, and 1 VSI-B). Bars, Excitatory synapses; circles, inhibitory synapses; combinations of bars and circles, multicomponent synaptic potentials. Solid lines, Monosynaptic connections; dashed lines, polysynaptic pathways, with the intervening interneurons yet to be identified. $B$, Firing responses of three of the swim CPG neurons during a three-cycle swim motor program elicited by a $2 \mathrm{sec}, 10 \mathrm{~Hz}$ stimulus to pedal nerve 3 . Note that DSI firing continues long after the end of the motor program, whereas VSI-B and C2 are silent before and after.

Locomotion

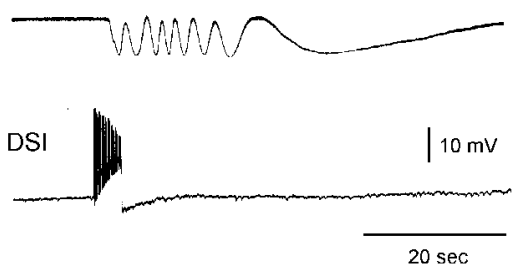

Figure 2. DSI activity elicits crawling. This experiment used an intact animal treadmill electrophysiology preparation, in which ciliary locomotion caused a drum positioned beneath the animal's foot to rotate. Sufficient depolarizing current was injected into a single DSI to elicit several seconds of firing (bottom trace). This produced a period of crawling that outlasted the DSI train by tens of seconds (top trace). With the treadmill apparatus, crawling, which is steady and nonrhythmic, is transduced into an oscillating voltage signal. The distance between two consecutive peaks represents $2 \mathrm{~mm}$ of locomotion. The maximum rate of treadmill turning corresponded to $1 \mathrm{~mm} / \mathrm{sec}$ of locomotion.

DSIs excite both of these neurons in normal saline (Popescu and Willows, 1999). We confirmed these results (Pd21: Fig. 3A, 10 preparations; Pd5: Fig. 3B, 4 preparations), and also tested whether these functionally excitatory connections persisted in a high divalent cation solution shown previously to suppress polysynaptic connectivity. We found that the DSIs connect to the contralateral $\operatorname{Pd} 21$ (Fig. 3C; three preparations) and Pd5 cilia neurons (Fig. 3D; three preparations) with direct, constant-latency, one-for-one EPSPs. In one preparation, all three DSIs on one side of the brain (DSI A-C) were tested and found to make direct connections onto the contralateral $\mathrm{Pd} 21$ and $\operatorname{Pd} 5$ neurons. No connections were observed between the DSIs and the ipsilateral $\operatorname{Pd} 21$ and $\operatorname{Pd} 5$ neurons.

\section{DSI firing correlates with both crawling and cilia neuron activity}

The treadmill experiment showed that directly activating a single DSI elicits crawling. We also tested the hypothesis that postswim
DSI firing (Fig. $1 B$ ) mediates crawling by comparing the duration of crawling, DSI firing, and cilia neuron firing. If the DSIs drive crawling, their firing should correspond to both cilia neuron firing and crawling behavior.

\section{Behavior}

Although it has been noted previously that Tritonia crawl after they swim (Audesirk, 1978b; Audesirk and Audesirk, 1980), the duration of this response had not been specifically determined. To measure this, 20 animals were transferred 5 at a time to a test arena, and the crawling status ("crawling" or "not crawling") of each animal was determined once every $5 \mathrm{~min}$ for $6 \mathrm{hr}$ (see Materials and Methods). For the first $3 \mathrm{hr}$, animals were left undisturbed, during which time crawling stimulated by the transfer gradually declined to a low level (Fig. $4 A$, left half). At the 3 -hr time point, all animals were made to swim by squirting $2 \mathrm{ml}$ of a $4 \mathrm{M} \mathrm{NaCl}$ solution onto their dorsal skin. The swims were followed by enhanced crawling, which declined gradually over time. Paired $t$ tests showed that significantly enhanced crawling lasted $\sim 90 \mathrm{~min}$ (Fig. $4 A ; p=0.0074$; Bonferroni-adjusted criterion of significance, $p<0.0083)$.

\section{Neuronal correlates}

DSIs. A minimum of $3 \mathrm{hr}$ after any previous swim motor program, the DSI spontaneous firing rate was recorded before and after a swim motor program elicited by a brief stimulus applied to PdN3 $(10 \mathrm{~Hz}, 2 \mathrm{sec}, 5 \mathrm{msec}$ pulses $)$. An ANOVA revealed a significant effect of nerve shock on DSI firing rate $\left(F_{(8,80)}=47.5 ; p<0.001\right)$. The DSI firing rate increased from $5 \pm 2$ spikes per minute just before to a peak rate of $111 \pm 10$ spikes per minute $(1.9 \mathrm{~Hz})$ during the first $30 \mathrm{sec}$ after the swim motor program, after which it progressively declined back to baseline. Post hoc testing indicated that DSI firing was significantly higher for 55 min after the motor program (Fig. 4B,E,F; $p<0.05$; 11 cells, eight preparations). Postswim elevated DSI firing was also observed after salt-elicited swims in intact animal electrophysiology prepara- 
A

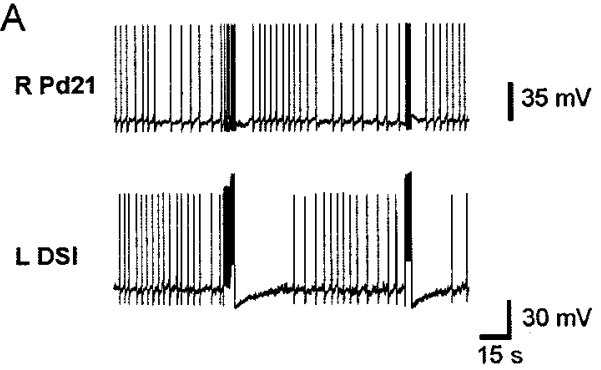

C

Figure 3. The DSIs directly excite the Pd21 and $\mathrm{Pd} 5$ cilia neurons. $A$, A DSI train evoked by intracellular stimulation produced excitation of the contralateral Pd21 in normal saline. $B$, Same result for Pd5. In this case, the duration of DSI stimulation is indicated by a bar. $C$, DSI produced constant latency, one-forone EPSPs in Pd21 in high divalent cation saline. $D$, Same result for Pd5. $R$, Right; $L$, left.

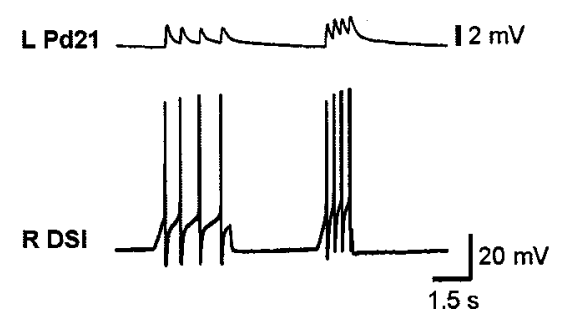

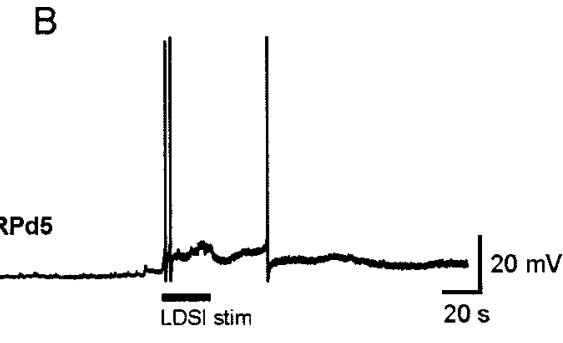

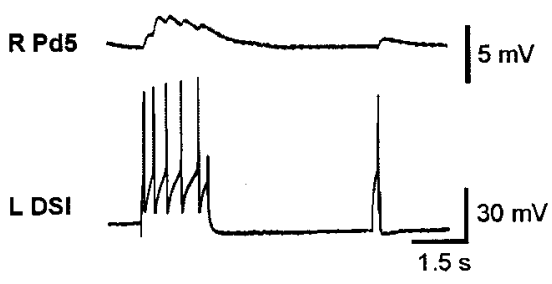

tions, but the duration of the effect was not determined in those experiments (four preparations).

Cilia neurons. The spontaneous firing rates of $\mathrm{Pd} 21$ and $\mathrm{Pd} 5$ also underwent a long-lasting increase after the swim motor program. An ANOVA revealed a significant effect of nerve shock on Pd21 firing rate $\left(F_{(6,24)}=18.5 ; p<0.001\right)$. Post hoc testing indicated that the firing rate of $\mathrm{Pd} 21$ was significantly increased for $75 \mathrm{~min}$ (Fig. 4C,E; $p<0.05$; five cells, four preparations). An ANOVA also demonstrated a significant effect of nerve shock on Pd5 firing rate $\left(F_{(6,36)}=9.96 ; p<0.001\right)$. Post hoc testing indicated that Pd5 firing was significantly increased for $35 \mathrm{~min}$ (Fig. 4D,F; $p<0.05$; seven cells, four preparations).

\section{Low rates of DSI stimulation activate the foot cilia}

To test whether the recorded rates of post-swim motor program DSI activity were sufficient to activate the locomotory cilia, we used a semi-intact preparation in which individual DSIs could be driven intracellularly while monitoring the movement of charcoal particles applied to the contralateral foot. In all instances, cilia that had been stationary before DSI stimulation (particles moving at $<0.5 \mathrm{~mm} / \mathrm{min}$ ) became active (particles moving at $>2.25$ $\mathrm{mm} / \mathrm{min}$ ) when single DSIs were driven at $2 \mathrm{~Hz}$ (five trials in three preparations), $1 \mathrm{~Hz}$ (five trials in two preparations), and 0.5 $\mathrm{Hz}$ (four trials in two preparations). The average latency to movement onset was on the order of minutes and depended on the stimulation rate: $0.5 \mathrm{~Hz}=5.0 \mathrm{~min} ; 1 \mathrm{~Hz}=2.6 \mathrm{~min}$; and 2 $\mathrm{Hz}=1.0 \mathrm{~min}$. In all cases, charcoal particle transport continued for tens of seconds after the cessation of DSI stimulation.

\section{Tonic DSI firing actively maintains the elevated cilia neuron firing after the swim motor program}

The above observations support the hypothesis that after a swim, elevated DSI firing acts to increase the tonic firing rate in the cilia neurons, resulting in enhanced crawling. To more directly test this idea, we next examined the effect of transiently removing this postswim DSI input to the cilia neurons.

In isolated brain preparations, a $\mathrm{Pd} 21$ and two or three of its three contralateral DSIs were simultaneously impaled with intracellular electrodes. After a rest period, a nerve stimulus was delivered to PdN3 to elicit the swim motor program, resulting in the usual elevated tonic firing in both neuron types. During this period of enhanced firing, a hyperpolarizing current was simultaneously injected into all impaled DSIs to suppress their firing for several seconds. This was found to reduce or eliminate the tonic firing in the $\mathrm{Pd} 21$ neurons (Fig. $5 A$; three $\mathrm{Pd} 21$ cells, two preparations). In the one instance in which it was tested, suppressing the firing of all three ipsilateral DSIs also eliminated the enhanced post-swim motor program firing in the contralateral Pd5 (Fig. 5B). The DSIs are not electrically coupled to either $\mathrm{Pd} 21$ or Pd5; thus the effect of suppressing DSI firing on the cilia neuron firing rate is not attributable to the spread of the hyperpolarizing current. These results are consistent with a role for the DSIs in actively maintaining the post-swim motor program elevated cilia neuron firing rate.

Prolonged DSI firing also occurs in response to stimuli too weak to trigger the swim motor program

Although Tritonia crawl after they swim, they also crawl in other contexts, such as in response to water flow (Willows, 1978; Murray et al., 1992), weak salt (Audesirk, 1978b), or mechanical stimulation, e.g., being picked up or rolled (Fig. 4A, left half). As a first step in testing whether DSI activity might mediate these other instances of locomotion, we next tested whether stimuli too weak to elicit the swim motor program would also elicit prolonged DSI firing.

We found that nerve stimuli below the threshold for eliciting the swim motor program produced prolonged enhanced DSI firing in the isolated brain preparation. An ANOVA revealed a significant effect of weak nerve shock on DSI firing rate $\left(F_{(11,44)}=\right.$ 28.5; $p<0.001)$. Post hoc testing indicated that the DSI firing rate was significantly higher for at least $10 \mathrm{~min}$ (Fig. 6 ; $p<0.05$; five cells, three preparations). Longer recordings were not made, so the duration of this effect is not known. This result is consistent with the hypothesis that the multifunctional swim network mediates crawling in contexts other than swimming (see Discussion).

\section{All members of the swim CPG make direct or indirect connections to the cilia neurons}

Previous studies reported that CPG neuron C2 makes direct, mixed excitatory and inhibitory synaptic connections to both $\mathrm{Pd} 21$ and Pd5 (Snow, 1982), and that C2 trains elicit action potential trains in Pd21 (Audesirk, 1978b; Snow, 1982). After confirming 
A

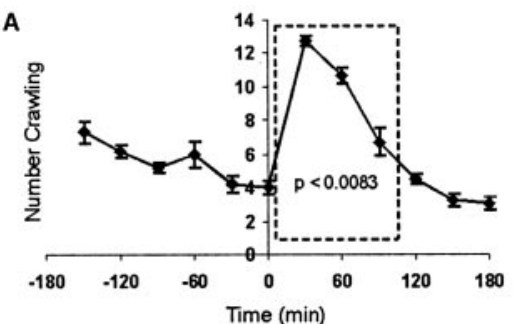

C

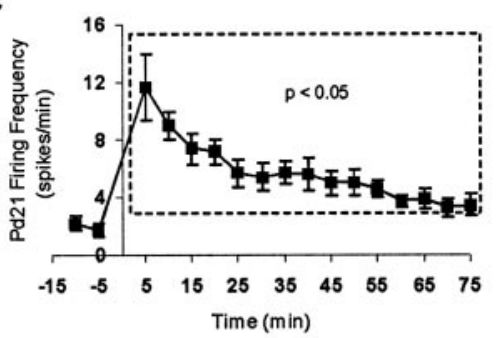

B

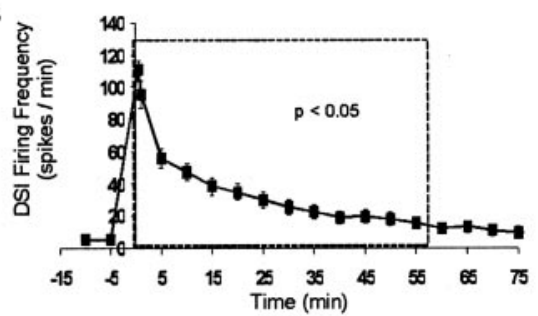

D

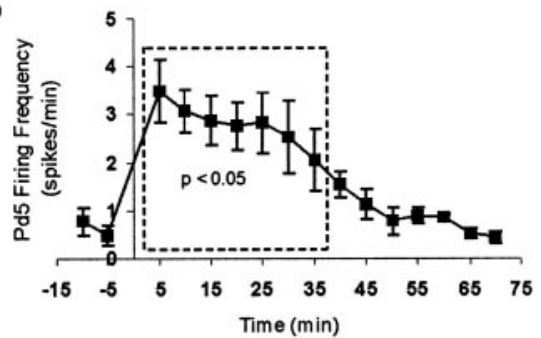

E

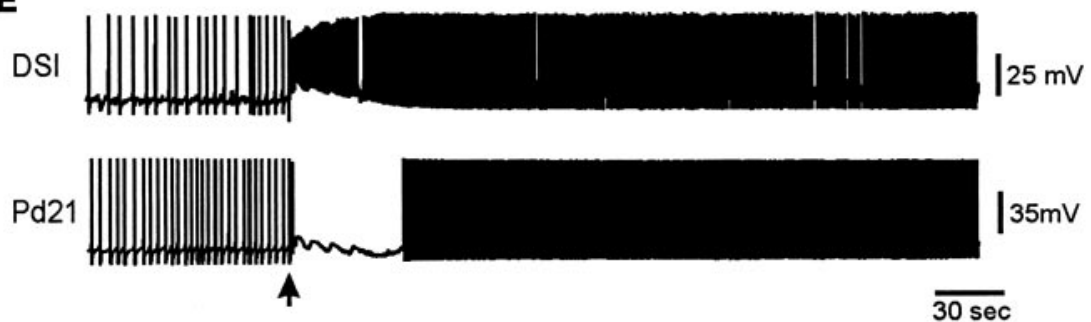

$\mathbf{F}$

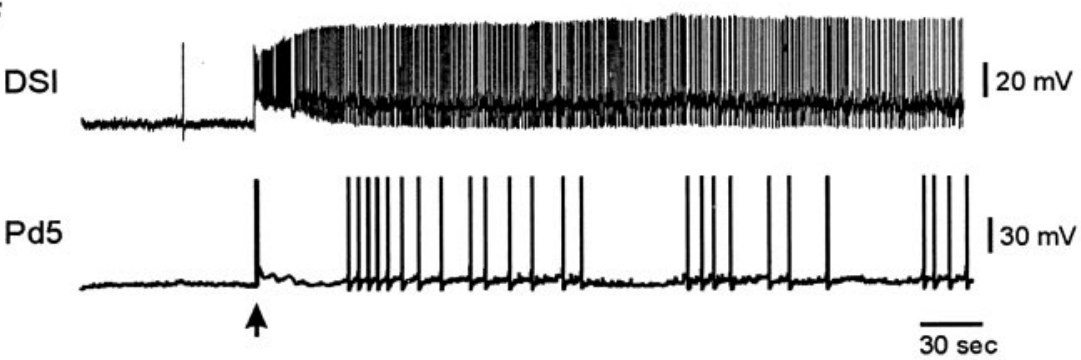

Figure 4. DSI firing correlates with crawling and cilia neuron activity. $A$, Duration of postswim crawling in freely behaving animals. Twenty animals were transferred to a test arena, and their crawling was assessed once every $5 \mathrm{~min}$ for $6 \mathrm{hr}$. Each point represents the mean of the six determinations of how many were crawling during the corresponding $30 \mathrm{~min}$ period. The transfer itself stimulated crawling, which declined over the first $3 \mathrm{hr}$. At that point, all animals were made to swim (time 0 ). For statistical analysis, each of the six postswim means was compared with the final pretest mean. The swim was followed by significantly enhanced crawling lasting $90 \mathrm{~min}$. The dotted boxes in this and the subsequent graphs represent the duration of the significant effect. At 5 min after the swim, all animals that had their foot in contact with the substrate were crawling (13 of 13 animals). $B$, Duration of enhanced DSI firing after a swim motor program in the isolated brain. The motor program was elicited at time 0 . DSI firing was significantly enhanced for $55 \mathrm{~min}$ (14 cells, 10 preparations). $C$, Duration of enhanced Pd21 firing. Pd21 firing was significantly enhanced for 75 min (8 cells, 7 preparations). $D$, Duration of enhanced Pd5 firing. Pd5 firing was significantly enhanced for $35 \mathrm{~min}$ (7 cells, 4 preparations). E, Example of DSI and Pd21 firing before and after a nerve stimulus-elicited swim motor program. Arrows in $E$ and $F$ indicate the time of the nerve stimulus. The individual cycles of the motor program cannot be clearly seen in the DSI trace at this time base but are visible as voltage oscillations in Pd21.F, Example of DSI and Pd5 firing before and after a nerve stimulus-elicited swim motor program. The statistics for $B-D$ were only applied to the cells (numbers listed in Results) that were recorded for the full period shown on the graphs. the DSI connection to the cilia neurons (Fig. 3), we became interested in the possibility that the entire swim CPG might be wired to exert a controlling function over crawling. We therefore tested the remaining two CPG neurons, ventral swim interneuron A (VSI-A) and VSI-B. Stimulation of VSI-A produced one-forone, constant-latency IPSPs in the contralateral Pd5 (Fig. 7A) and $\operatorname{Pd} 21$ (Fig. $7 B$ ) in both normal and high divalent cation saline (three preparations each). This inhibition was strong enough that VSI-A stimulation significantly reduced the elevated firing of $\operatorname{Pd} 21$ observed after a swim (Fig. $7 C$; four preparations).

Stimulating CPG neuron VSI-B also reduced the elevated post-swim motor program firing of $\mathrm{Pd} 21$ (Fig. 8A). However, when tested in high divalent cation saline, this connection was clearly not one-for-one (Fig. $8 B$; four preparations), indicating that it is polysynaptic, involving the recruitment of an as yet unidentified inhibitory interneuron. We also tested for, but did not observe, a connection between VSI-B and the contralateral Pd5 (two preparations). In summary, every member of the swim CPG network was found to make either direct (DSI, C2, and VSI-A) or indirect (VSI-B) connections with one or both of the cilia neurons $\operatorname{Pd} 21$ and $\operatorname{Pd} 5$ (Fig. $1 A$ ), indicating that the previously described swim network is wired appropriately to mediate both swimming and the excitatory and inhibitory control of crawling.

What are the functions of the non-DSI connections from the swim CPG to the cilia neurons? These neurons either undergo no apparent long-lasting change in spontaneous firing rate after swimming (VSI-A) or are silent when not swimming (C2 and VSI-B; Fig. 1B). A possible function for the inhibitory connections (VSI-A and VSI-B) might be to mediate touch-induced inhibition of crawling. A previous report (Audesirk, 1978b) observed that tactile stimulation of the animal's anterior end caused both locomotion and ciliary beating to stop and produced hyperpolarizing inhibitory input to the Pd21 neurons. Using a semiintact electrophysiology preparation, we found that stimulating the skin with a glass probe elicited VSI-B action potentials in three of four preparations (Fig. 9). Because these neurons inhibit both the DSIs (Getting, 1983b) and Pd21 (Fig. 8), they seem well suited to mediate touch-induced inhibition of crawling. Additional work is needed to adequately test this hypothesis. Tactile stimulation produced no consistent effects, either excitatory or inhibitory, in VSI-A (L.-M. Tian, T. A. Hoppe, and W. N. Frost, unpublished results). It may be that the excitatory tactile receptive field of VSI-A has yet to be found, or that this interneuron 

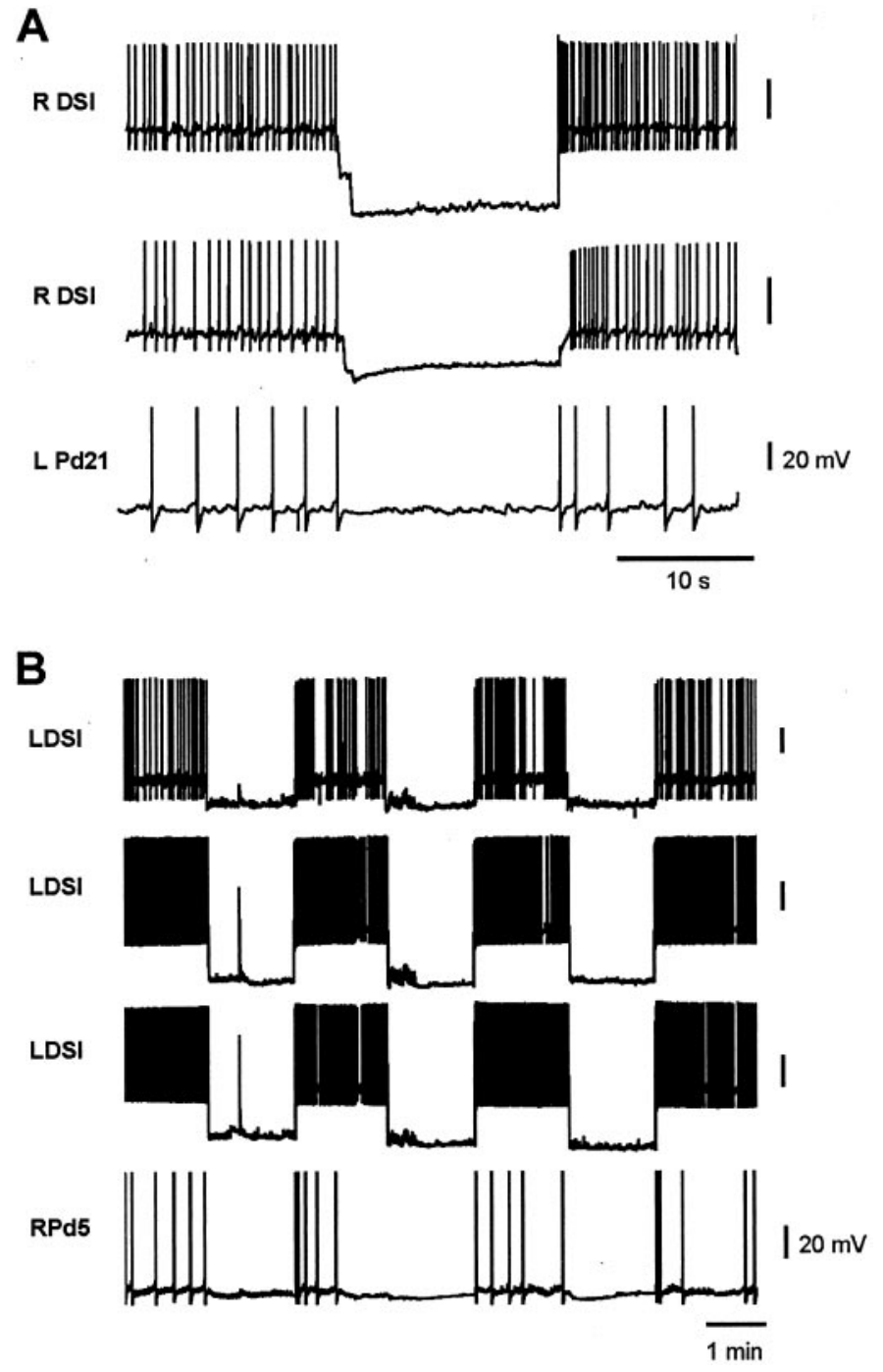

Figure 5. Tonic DSI firing actively maintains the elevated cilia neuron firing that follows the swim motor program. A, After a swim motor program (data not shown), the DSIs and cilia neurons fire at an elevated tonic rate. Hyperpolarizing two of the three contralateral DSIs during this period acted to eliminate the tonic firing in Pd21. $B$, In a similar experiment, hyperpolarizing all three contralateral DSIs repeatedly reduced or eliminated the elevated tonic firing in Pd5. $R$, Right; $L$, left.

may inhibit crawling in other behavioral contexts, such as when Tritonia encounter food (Audesirk, 1978b).

\section{DISCUSSION}

\section{The multifunctional Tritonia network}

Previous work suggested that the Tritonia escape swim network mediates two different behaviors. Weak-to-moderate stimuli were proposed to elicit withdrawal responses, whereas strongly aversive stimuli reconfigure the network into its swim pattern-generating mode (Getting and Dekin, 1985a,b). Although its withdrawal function remains mostly untested experimentally, its role in the swim is well established (Getting, 1983a). Here we report that this same network also appears to control a third, very different behavior, cilia-mediated crawling. Several observations support this conclusion. First, the DSI neurons of the swim CPG directly excite the cilia neurons $\mathrm{Pd} 21$ and $\mathrm{Pd} 5$, which elicit crawling (Fig. 3 ). These connections are sufficiently strong that intracellular stimulation of a single DSI elicits crawling in intact animal treadmill preparations (Fig. 2). Second, for nearly $1 \mathrm{hr}$ after a swim motor program, the DSIs fire at an elevated rate (Fig. 4B). This duration correlates reasonably well with the period freely behaving animals crawl after they swim (Fig. $4 A$ ). Third, driving single DSIs at rates as low as $0.5 \mathrm{~Hz}$ activates cilia-mediated transport of carbon particles on the foot. In our recordings, all six DSIs fired tonically at or above this level for nearly $25 \mathrm{~min}$ after the swim motor program (Fig. 4B), supporting the likelihood that the DSIs mediate the crawling observed during this period. Fourth, silencing the tonically active DSIs after a swim motor program substantially reduces or eliminates ongoing cilia neuron firing (Fig. 5), indicating that the DSIs are major contributors to the synaptic input driving these cells. Finally, all of the other swim CPG neurons (C2, VSI-A, and VSI-B) also connect to the cilia neurons, two of the three monosynaptically (Figs. 7, 8). Taken together, these observations indicate that the Tritonia swim CPG network appears to be wired to mediate excitatory as well as inhibitory control of crawling. Given that the animals spend significantly more time crawling than swimming, it seems possible that the control of crawling may actually be the predominant operating mode of the network.

Multifunctional networks are frequently found to mediate closely related behaviors that use the same or similar muscle groups. The present example is a striking exception. In Tritonia, swimming is a brief, rhythmic behavior mediated by alternating ventral and dorsal whole-body muscular contractions. Postswim crawling, on the other hand, is two orders of magnitude longer lasting, nonrhythmic, and cilia-mediated. To our knowledge, this is the first direct demonstration of multifunctional network control over muscular and ciliary effector systems. Similar control has been proposed for swimming and ciliary crawling by the As1-4 neurons, homologs of the DSIs, in the marine mollusk Pleurobranchaea (Jing and Gillette, 2000). Another example may be the control of respiration and ciliary crawling by the L/RpeD11 interneurons in the freshwater snail Lymnaea (Syed and Winlow, 1989, 1991). Multifunctional network control of muscular and ciliary effector systems may also occur in vertebrates. Stimulation of respiratory c-fibers accelerates airway mucociliary beat frequency (Lindberg and Mercke, 1986; Lindberg et al., 1987), inhibits the muscular control of breathing (Wang et al., 1996), and may elicit coughing (Coleridge and Coleridge, 1994).

An additional difference between these two Tritonia behaviors concerns their degree of flexibility. Swimming is a stereotyped, nondirectional behavior that is relatively unaffected by sensory feedback once under way. Crawling, on the other hand, varies in

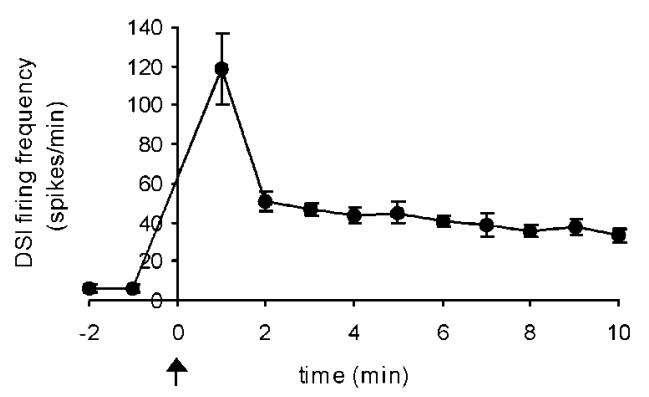

Figure 6. Stimuli subthreshold for producing the swim nonetheless cause long-lasting DSI firing. A weak nerve stimulus administered to PdN3 at the arrow $(10 \mathrm{~Hz}, 2 \mathrm{sec})$ elevated the rate of spontaneous DSI firing for at least $10 \mathrm{~min}$. 


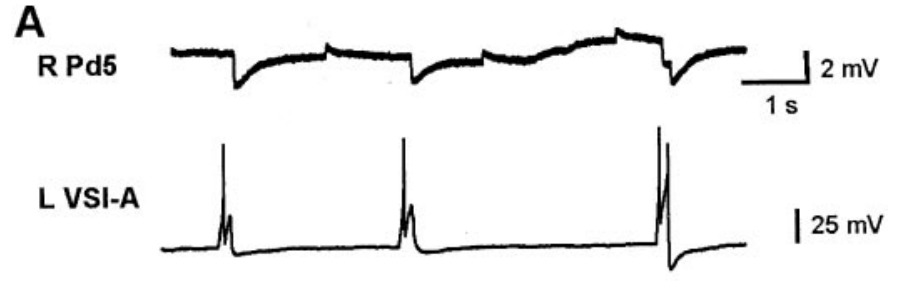

B
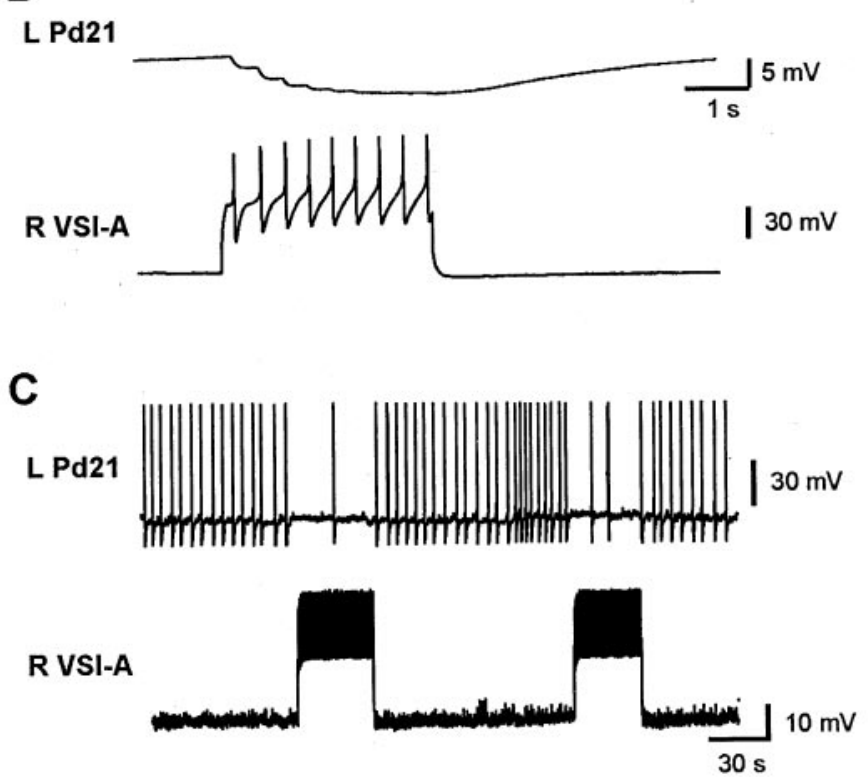

Figure 7. CPG neuron VSI-A monosynaptically inhibits the cilia neurons. $A$, VSI-A action potentials elicited unitary, one-for-one IPSPs in the contralateral $\mathrm{Pd} 5$ in high divalent cation saline. The third current pulse caused two action potentials and two corresponding IPSPs in Pd5. B, In high divalent cation saline, a train of VSI-A spikes elicited one-for-one, summating IPSPs in the contralateral Pd21. $C$, The inhibitory VSI-A connection to $\mathrm{Pd} 21$ was sufficient to inhibit P21 firing in normal saline. VSI-A was driven via the intracellular electrode to fire two trains of action potentials. The slight depolarization of Pd21 during the inhibition was attributable to a reversal of the IPSP, which had initially been hyperpolarizing when Pd21 was penetrated. $R$, Right; $L$, left.

speed and direction and is readily modified by sensory input, inhibited when the animal's oral veil comes into contact with food or tactile stimuli (Audesirk, 1978b; Audesirk and Audesirk, 1980) and excited by salt stimuli too weak to elicit the swim (Audesirk, 1978b). Our results support the possibility that the network also mediates such stimulus-elicited alterations of ongoing crawling. For example, the C2 and VSI-B neurons are silent before and after the swim motor program (Fig. $1 B$ ); hence, they play no role in postswim crawling. They do, however, display brief firing responses to different skin stimuli (Fig. 9; Tian and Frost, unpublished results). Given their respective excitatory and inhibitory effects on the cilia neurons, these neurons thus seem well suited to mediate the effects of different stimuli on ongoing crawling.

Many studies have described multifunctional networks that mediate motor programs underlying different, muscle-based behaviors (Getting, 1989; Dickinson, 1995; Marder and Calabrese, 1996). For example, in the crustacean stomatogastric nervous system, a relatively small number of neurons generate multiple motor programs involved in the grinding and transport of food (Dickinson et al., 1990; Meyrand et al., 1991; Weimann and Marder, 1994; Nusbaum et al., 2001). In the cockroach, a pair of
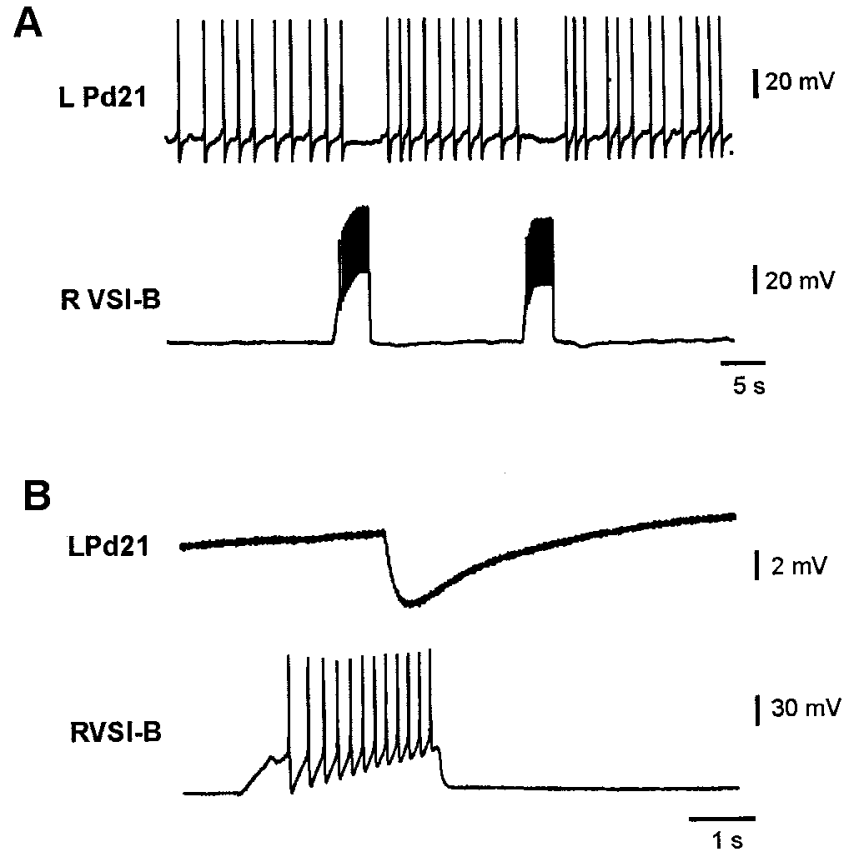

Figure 8. CPG neuron VSI-B polysynaptically inhibits Pd21. A, Driving VSI-B caused a decrease in the firing rate of the contralateral Pd21. $B$, In high divalent cation saline, a train of VSI-B spikes recruited IPSPs into $\mathrm{Pd} 21$ in a non-one-for-one manner, indicating that this inhibitory connection is indirect. $L$, Left; $R$, right.

giant interneurons trigger both flight and running, depending on the behavioral context (Ritzmann et al., 1980). In the stick insect, a multifunctional interneuronal network has been suggested to underlie leg movements involved in tactile reflexes, rocking, and walking (Kittmann et al., 1996). During flight, interneuronal members of the locust respiratory oscillator are reconfigured to fire in phase with the flight rhythm (Ramirez, 1998). Finally, a single arterial shortening neuron participates in six different behaviors in the marine mollusk Aplysia (Xin et al., 1996). Multifunctional neurons have been proposed to participate in diverse behaviors in vertebrates as well (Dickinson, 1995), including the control of licking, swallowing, and the rejection of food (Travers et al., 2000) and the control of both respiration and vomiting (Zheng et al., 1997).

Taken together, the present results and previous studies support the view that the Tritonia swim CPG may be involved in the production of a highly diverse set of behaviors, including (1) reflexive withdrawal (Getting and Dekin, 1985a), (2) swimming

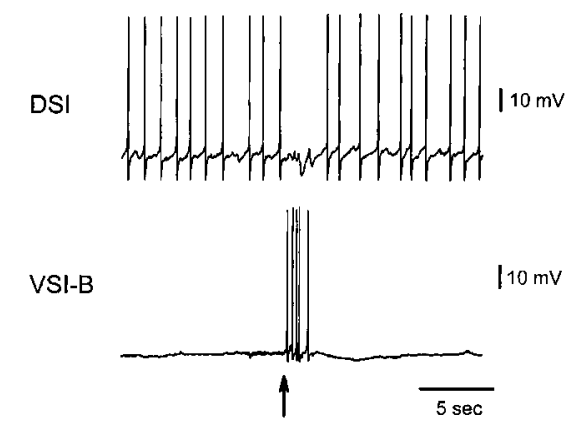

Figure 9. Response of DSI and VSI-B to tactile skin stimulation. Poking the skin with a glass probe (arrow) elicited action potentials in VSI-B and inhibition in DSI. 
(Getting, 1983a), (3) postswim crawling, (4) stimulus-elicited inhibition of ongoing crawling, and (5) stimulus-elicited acceleration of ongoing crawling. The available data most strongly support roles for the network in swimming and postswim crawling. Additional work is needed to rigorously test the other hypothesized functions, as well as to determine whether the network also mediates crawling in other contexts, such as during food seeking (Willows, 1978), and during the animal's positive rheotaxis to water flow (Field and MacMillan, 1973; Willows, 1978; Murray et al., 1992). At present, the members of the swim CPG represent the only identified interneuronal inputs to the cilia neurons. However, crawling is a more flexible behavior than swimming, with a much broader range of speed, duration, and controlling stimuli. From this perspective, it seems possible that the complete crawling network will be found to include interneurons located outside the swim CPG, involving a more distributed network organization.

\section{Additional features}

Although multifunctionality has the advantage of neural economy, it has inherent problems as a design strategy. How, for example, do the different functional subcircuits of anatomically superimposed networks operate without interfering with one another? In this Tritonia example, the two motor programs are coactive during the swim, when the DSIs excite the efferent neuron populations for both swimming and crawling. There is no behavioral conflict, however, because although the foot cilia are active during the swim (Audesirk, 1978b), crawling cannot occur while the foot is out of contact with the substrate. One benefit of this coactivation may be to ensure that once the swim ends, crawling commences at full speed as soon as the animal reestablishes foot contact with the substrate.

In Tritonia, there is an obvious survival advantage to having a single network mediate withdrawal, swimming, and crawling. Although withdrawal and crawling can occur in the absence of swimming, strongly aversive stimuli elicit all three behaviors in a defined temporal sequence. Having a common network control these functionally related but disparate behaviors would ensure the efficient coordination of this complex, integrated escape response. It seems reasonable to suppose that the network originally evolved to mediate crawling (and withdrawal) to a wide range of stimulus intensities, with its rhythm-generating ability added later. This hypothesis is supported by the fact that DSI homologs are present in opisthobranch species that do not swim (Katz et al., 2001). We speculate that in such species, the DSI homologs will be found to mediate crawling.

The neural mechanisms by which this multifunctional network abruptly reconfigures into its swim mode are emerging (see below; Getting and Dekin, 1985a; Katz et al., 1994; Katz and Frost, 1997). An interesting finding is that this transition can be evoked by simply increasing the rate of tonic intracellular stimulation of the DSI neurons. Low stimulation rates elicit crawling (Fig. 2), whereas higher rates reconfigure the network to generate the rhythmic swim motor program (Fickbohm and Katz, 2000; Frost et al., 2001). This Tritonia version of a locomotor "gait change" may be analogous to that observed in the vertebrate mesencephalic locomotor region (Jordan, 1998), where a steady increase in the tonic extracellular stimulation rate triggers the locomotion circuitry to abruptly shift its output from trotting to rhythmic galloping (Shik et al., 1966). Although it is not known whether a single multifunctional network mediates this gait change in ver- tebrates, our present results are at least consistent with such a possibility.

\section{Neuromodulation in the network}

The role of the DSIs in network function is multifaceted. In addition to their conventional excitatory synaptic connections in the swim-crawling circuitry, these serotonergic interneurons produce a potent neuromodulatory enhancement of the excitability and synaptic connections of C2 (Katz et al., 1994; Katz and Frost, 1995a,b, 1997). Because the DSIs fire strongly at swim onset, and their neuromodulatory effects develop rapidly (within 2-3 sec), we have suggested previously that this intrinsic neuromodulation may play an important role in reconfiguring the resting network into its pattern-generating mode (Frost and Getting, 1989; Katz et al., 1994; Katz and Frost, 1997). Our present finding that DSI firing remains elevated for $1 \mathrm{hr}$ after an initial swim suggests that the neuromodulatory actions of these neurons may also persist long after the swim ceases. A possible behavioral consequence of such enduring modulation is supported by studies of nonassociative learning in Tritonia, which have found that an initial swim is followed by a 1-hr-long period of sensitization, during which subsequent swims have a significantly shorter onset latency (Brown et al., 1996; Frost et al., 1998; Mongeluzi and Frost, 2000). The similarity between the duration of DSI firing and latency sensitization supports the hypothesis (Katz et al., 1994; Frost et al., 1998) that persistent modulation produced by the postswim DSI firing serves to encode the memory for the sensitization.

Our results also have relevance for another issue regarding multifunctional networks, the degree to which they can incorporate modulation specific to one behavior without modifying the other behaviors mediated by the same network. As discussed, the persistent enhancement of DSI firing affects both the crawling and swimming functions of the network (its effect on withdrawal has not yet been tested). However, such conjoint effects need not always occur. For example, because C2 and VSI-B are silent during postswim crawling, learning-related changes specific to these neurons could affect subsequent swims without affecting ongoing crawling. Tritonia, because of its well characterized swimming and crawling circuitry, is an attractive model system in which to explore issues concerning the organization and operation of multifunctional networks.

\section{REFERENCES}

Audesirk G (1978a) Central neuronal control of cilia in Tritonia diomedea. Nature 272:541-543.

Audesirk G (1978b) Properties of central motor neurons exciting locomotory cilia in Tritonia diomedea. J Comp Physiol [A] 128:259-267.

Audesirk G, Audesirk T (1980) Complex mechanoreceptors in Tritonia diomedea. II. Neuronal correlates of a change in behavioral responsiveness. J Comp Physiol [A] 141:111-122.

Audesirk G, McCaman RE, Willows AOD (1979) The role of serotonin in the control of pedal ciliary activity by identified neurons in Tritonia diomedea. Comp Biochem Physiol C 62C:87-91.

Brown G, Frost WN, Getting PA (1996) Habituation and iterative enhancement of multiple components of the Tritonia swim response. Behav Neurosci 110:478-485.

Coleridge HM, Coleridge JC (1994) Pulmonary reflexes: neural mechanisms of pulmonary defense. Annu Rev Physiol 56:69-91.

Dickinson PS (1995) Interactions among neural networks for behavior. Curr Opin Neurobiol 5:792-798.

Dickinson PS, Mecsas C, Marder E (1990) Neuropeptide fusion of two motor pattern generator circuits. Nature 344:155-158.

Fickbohm DJ, Katz PS (2000) Paradoxical actions of the serotonin precursor 5-hydroxytryptophan on the activity of identified serotonergic neurons in a simple motor circuit. J Neurosci 20:1622-1634.

Field LH, MacMillan DL (1973) An electrophysiological and behavioral study of sensory responses in Tritonia (Gastropoda, Nudibranchia). Mar Behav Physiol 2:171-185.

Frost WN, Getting PA (1989) Self-tuning by a neural circuit: the DSI 
neurons of the Tritonia escape swim central pattern generator both modulate and mediate connections in the swim circuit. Soc Neurosci Abstr 15:1118.

Frost WN, Kandel ER (1995) Structure of the network mediating siphonelicited siphon withdrawal in Aplysia. J Neurophysiol 73:2413-2427.

Frost WN, Katz PS (1996a) Single neuron control over a complex motor program. Proc Natl Acad Sci USA 93:422-426.

Frost WN, Katz PS (1996b) Circuit modifications associated with reconfiguration of the Tritonia escape swim network by subthreshold stimuli. Soc Neurosci Abstr 22:2047.

Frost WN, Brandon CL, Mongeluzi DL (1998) Sensitization of the Tritonia escape swim. Neurobiol Learn Mem 69:126-135.

Frost WN, Hoppe TA, Wang J, Tian L-M (2001) Swim initiation neurons in Tritonia diomedea. Am Zool 41:952-961.

Getting PA (1983a) Neural control of swimming in Tritonia. Symp Soc Exp Biol 37:89-128.

Getting PA (1983b) Mechanisms of pattern generation underlying swimming in Tritonia. III. Intrinsic and synaptic mechanisms for delayed excitation. J Neurophysiol 49:1036-1050.

Getting PA (1989) Emerging principles governing the operation of neural networks. Annu Rev Neurosci 12:185-204.

Getting PA, Dekin MS (1985a) Tritonia swimming: a model system for integration within rhythmic motor systems. In: Model neural networks and behavior (Selverston AI, ed), pp 3-20. New York: Plenum.

Getting PA, Dekin MS (1985b) Mechanisms of pattern generation underlying swimming in Tritonia. IV. Gating of central pattern generator. J Neurophysiol 53:466-480.

Jing J, Gillette R (2000) Escape swim network interneurons have diverse roles in behavioral switching and putative arousal in Pleurobranchaea. J Neurophysiol 83:1346-1355.

Jordan LM (1998) Initiation of locomotion in mammals. Ann NY Acad Sci 860:83-93.

Katz PS, Frost WN (1995a) Intrinsic neuromodulation in the Tritonia swim CPG: serotonin mediates both neuromodulation and neurotransmission by the dorsal swim interneurons. J Neurophysiol 74:2281-2294.

Katz PS, Frost WN (1995b) Intrinsic neuromodulation in the Tritonia swim CPG: The serotonergic dorsal swim interneurons act presynaptically to enhance transmitter release from interneuron $\mathrm{C} 2$. J Neurosci 15:6035-6045.

Katz PS, Frost WN (1997) Removal of spike frequency adaptation via neuromodulation intrinsic to the Tritonia escape swim central pattern generator. J Neurosci 17:7703-7713.

Katz PS, Getting PA, Frost WN (1994) Dynamic neuromodulation of synaptic strength intrinsic to a central pattern generator circuit. Nature 367:729-731.

Katz PS, Fickbohm DJ, Lynn-Bullock CP (2001) Evidence that the central pattern generator for swimming in Tritonia arose from a nonrhythmic neuromodulatory arousal system: implications for the evolution of specialized behavior. Am Zool 41:962-975.

Kittmann R, Schmitz J, Buschges A (1996) Premotor interneurons in generation of adaptive leg reflexes and voluntary movements in stick insects. J Neurobiol 31:512-532.

Kupfermann I, Weiss KR (2001) Motor program selection in simple model systems. Curr Opin Neurobiol 11:673-677.

Lennard PR, Getting PA, Hume RI (1980) Central pattern generator mediating swimming in Tritonia. II. Initiation, maintenance, and termination. J Neurophysiol 44:165-173.

Lieske SP, Thoby-Brisson M, Telgkamp P, Ramirez JM (2000) Reconfiguration of the neural network controlling multiple breathing patterns: eupnea, sighs and gasps. Nat Neurosci 3:600-607.

Lindberg S, Mercke U (1986) Antidromic nerve stimulation accelerates mucociliary activity in rabbit maxillary sinus. Acta Otolaryngol 101:484-493.

Lindberg S, Dolata J, Mercke U (1987) Stimulation of C fibers by am- monia vapor triggers mucociliary defense reflex. Am Rev Respir Dis 135:1093-1098.

Mackie GO, Meech RW (1985) Separate sodium and calcium spikes in the same axon. Nature 313:791-793.

Marder E, Calabrese RL (1996) Principles of rhythmic motor pattern generation. Physiol Rev 76:687-717.

McClellan AD, Brown GD, Getting PA (1994) Modulation of swimming in Tritonia: excitatory and inhibitory effects of serotonin. J Comp Physiol [A] 174:257-266.

Meyrand P, Simmers J, Moulins M (1991) Construction of a patterngenerating circuit with neurons of different networks. Nature 351:60-63.

Mongeluzi DL, Frost WN (2000) Dishabituation of the Tritonia escape swim. Learn Mem 7:43-47.

Morton DW, Chiel HJ (1994) Neural architectures for adaptive behavior. Trends Neurosci 17:413-420.

Murray JA, Hewes RS, Willows AOD (1992) Water-flow sensitive pedal neurons in Tritonia: role in rheotaxis. J Comp Physiol [A] 171:373-385.

Nusbaum MP, Blitz DM, Swensen AM, Wood D, Marder E (2001) The roles of co-transmission in neural network modulation. Trends Neurosci 24:146-154.

Pearson KG (1993) Common principles of motor control in vertebrates and invertebrates. Annu Rev Neurosci 16:265-297.

Popescu IR, Willows AOD (1999) Sources of magnetic sensory input to identified neurons active during crawling in the marine mollusc Tritonia diomedea. J Exp Biol 202:3029-3036.

Ramirez JM (1998) Reconfiguration of the respiratory network at the onset of locust flight. J Neurophysiol 80:3137-3147.

Ritzmann RE, Tobias ML, Fourtner CR (1980) Flight activity initiated via giant interneurons of the cockroach: evidence for bifunctional trigger interneurons. Science 210:443-445.

Shik ML, Severin FV, Orlovsky GN (1966) Control of walking and running by means of electrical stimulation of the mid-brain. Biophysics 11:756-765.

Snow RW (1982) Characterization of the synaptic actions of an interneuron in the central nervous system of Tritonia. J Neurobiol 13:251-266.

Syed NI, Winlow W (1989) Morphology and electrophysiology of neurons innervating the ciliated locomotor epithelium in Lymnaea stagnalis. Comp Biochem Physiol A 93:633-644.

Syed NI, Winlow W (1991) Coordination of locomotor and cardiorespiratory networks of Lymnaea stagnalis by a pair of identified interneurones. J Exp Biol 158:37-62.

Travers JB, DiNardo LA, Karimnamazi H (2000) Medullary reticular formation activity during ingestion and rejection in the awake rat. Exp Brain Res 130:78-92.

Wang AL, Blackford TL, Lee LY (1996) Vagal bronchopulmonary $\mathrm{C}$-fibers and acute ventilatory response to inhaled irritants. Respir Physiol 104:231-239.

Weimann JM, Marder E (1994) Switching neurons are integral members of multiple oscillatory networks. Curr Biol 4:896-902.

Willows AO, Pavlova GA, Phillips NE (1997) Modulation of ciliary beat frequency by neuropeptides from identified molluscan neurons. J Exp Biol 200:1433-1439.

Willows AOD (1978) Physiology of feeding in Tritonia. I. Behavior and mechanics. Mar Behav Physiol 5:115-135.

Willows AOD, Dorsett DA, Hoyle G (1973) The neuronal basis of behavior in Tritonia. I. Functional organization of the central nervous system. J Neurobiol 4:207-237.

Xin Y, Weiss KR, Kupfermann I (1996) An identified interneuron contributes to aspects of six different behaviors in Aplysia. J Neurosci 16:5266-5279.

Zheng Y, Umezaki T, Nakazawa K, Miller AD (1997) Role of preinspiratory neurons in vestibular and laryngeal reflexes and in swallowing and vomiting. Neurosci Lett 225:161-164. 